\title{
GRANDES CONTROVERSIAS DE LA HISTORIA DE LA CIENCIA ECONÓMICA: BÖHM-BAWERK REFUTA LA TEORÍA MARXISTA DE LA EXPLOTACIÓN
}

JOSÉ IGNACIO DEL CASTILLO*

En la primera mitad del siglo XIX el liberalismo reina triunfante en Occidente. Se trata de un movimiento de emancipación, enemigo de los privilegios que a través del estado y mediante los impuestos y las restricciones a la libertad económica se reservan unas clases sociales - nobleza, clero y gremios- a expensas del resto de la población. El liberalismo opone la razón y la ciencia frente al oscurantismo y la superstición. En el campo de la economía, el liberalismo tiene su expresión en la defensa del laissez faire frente al mercantilismo. Adam Smith primero y David Ricardo después, ya han establecido las bases de la que hoy se conoce como Escuela Clásica de Economía. El sistema de Ricardo aunque adolece de graves fallos, aparenta ser un edificio de construcción lógica impecable, lo que impresiona notablemente a sus contemporáneos.

Paralelamente y además de los reaccionarios partidarios del Antiguo Régimen, existe un movimiento socialista utópico, acientífico y cuasi-místico cuyos principales representantes son Fourier, Owen y Saint Simon y junto a él uno algo más fundamentado aunque no mucho más con Lasalle, Sismondi y Roedbertus. En su Historia del Pensamiento Económico, Murray Rothbard hace un formidable repaso genealógico de este movimiento que abarcaría desde Espartaco a Tomás Moro, de Campanella a Thomas Munzer y los anabaptistas alemanes y de Platón o Esparta hasta Gracus Babeuf y su Liga de los Iguales.

* Instituto Juan de Mariana. 
Es en este contexto histórico en el que aparece Karl Marx. Marx había alcanzado notoriedad con la publicación en 1848 del Manifiesto Comunista, pero es en 1857 con El Capital cuando reivindica su lugar dentro de la Ciencia Económica. Lo que caracterizaba a Marx frente al resto de socialistas utópicos era su argumentación científica (pseudo-científica en realidad) y su lenguaje «liberal» para atacar el liberalismo. Marx sostenía que también él quería acabar con los privilegios de clase y con el estado como instrumento de explotación. Igual que los liberales se definía como progresista, racional. No sólo eso. Los liberales eran la derecha. El sistema de laissez faire era una nueva forma de opresión. En el mismo, una clase: los propietarios capitalistas y burgueses explotaba a otra: los trabajadores asalariados por él denominados proletariado. Así como la nobleza vivía de los tributos procedentes del resto de la sociedad y así como los señores feudales se alimentaban del trabajo de los siervos de la gleba, según Marx los capitalistas vivían merced al beneficio empresarial que no podía provenir de otro lado que del excedente sustraído al trabajador al que denominó plusvalía. A partir de ahí Marx cimentó sus conclusiones sobre el futuro del capitalismo: creciente concentración de riqueza en pocas manos y tendencia al monopolio - la eterna cantinela de pobres más pobres y ricos más ricos-, tasa de beneficio decreciente conforme aumenta la acumulación de capital con las consiguientes crisis de cada vez mayor intensidad, para desembocar finalmente en una dictadura del proletariado cuando los desposeídos cada vez mayores en número se apoderasen de la propiedad capitalista.

La acusación era gravísima y la teoría tan tremendamente ambiciosa como intento de explicar la realidad como para ser ignorada. Se hacía por tanto ineludible un examen de la misma en profundidad, pues de su veracidad o falsedad podía depender el futuro de la humanidad. El insigne economista austríaco Eugen von Böhm-Bawerk (1850-1914) se dedicó a tan esencial cometido con el resultado que vemos a continuación. 
I

\section{LA TEORÍA DE LA EXPLOTACIÓN Y SU REFUTACIÓN}

Con el fin de no hacer excesivamente prolija la exposición, he optado por ir simultaneando la argumentación marxista contenida en el primer volumen de El Capital con la refutación de Böhm-Bawerk incluida en el capítulo dedicado a La Teoría de la Explotación dentro de su monumental Historia y crítica de las teorías del interés que es el primer volumen de la obra Capital e Interés. La controversia tiene dos partes como veremos, pues incluso el propio Marx detectó contradicciones en su sistema. Marx prometió resolverlas en el tercer volumen de El Capital. Tras la publicación de este tercer volumen, Böhm-Bawerk examinó dicha «solución» en su Conclusión del sistema marxiano.

II

EL PRIMER VOLUMEN DE EL CAPITAL Y LA CRÍTICA DE BÖHM-BAWERK

Marx comienza a construir su teoría invocando la autoridad de Aristóteles: «No puede existir cambio sin igualdad, ni igualdad sin conmensurabilidad». Por tanto, según Marx en las dos cosas intercambiadas tiene que existir «un algo común y de la misma magnitud».

Aquí Bóhm-Bawerk detecta la primera falsedad: En realidad el valor no es intrínseco a las cosas, sino algo subjetivamente apreciado por cada individuo según su situación y necesidades. En efecto, en un intercambio ambas partes valoran en menor medida lo que ceden que lo que obtienen. Para poner a prueba la teoría marxista, Jim Cox planteaba la siguiente pregunta: ¿Cuántas veces ha ido el lector al mercado a cambiar un billete de un dólar por otro billete idéntico y luego otra vez y otra,...? Desgraciadamente, la teoría de la igualdad de valor intrínseco de las cosas intecambiadas es pilar básico tanto de la terrible teoría mercantilista según la cual en el intercambio si alguien gana es porque el otro pierde, como en el no menos pernicioso movimiento contemporáneo que denuncia el «comercio injusto» NorteSur. 
Un estudiante de lógica sabe que cualquier conclusión obtenida a partir de una premisa falsa o de un razonamiento falaz carece de valor científico. Pero no es que Marx deduzca coherentemente todo su sistema a partir de esta única falsedad, es que los errores y las falacias se multiplican en cada paso. Seguimos.

Para investigar ese «algo común» característico del valor de cambio, Marx repasa las diversas cualidades que poseen los objetos equiparados por medio del cambio. Eliminando y excluyendo aquellas que no resisten la prueba, se queda sólo con una que según él, sí pasa el examen: «ser productos del trabajo».

Sin embargo Marx hace trampa y Böhm-Bawerk lo evidencia. En primer lugar es falso que todos los bienes intercambiados sean producto del trabajo. Por ejemplo, los recursos naturales tienen valor y son intercambiados, pero no son producto de ningún trabajo.

Certeramente objeta Knies a Marx: «Dentro de la exposición de Marx no se ve absolutamente ninguna razón para que la igualdad expresada en la fórmula: 1 libra de trigo= x quintales de madera producidos en el bosque no sea sustituida con igual derecho por esta otra: 1 libra de trigo $=x$ quintales de madera silvestre $=y$ yugadas de tierra virgen $=z$ yugadas de pastos naturales».

Pero no sólo eso. Es falso que esa sea la única característica común que pueda encontrarse en los bienes que son objeto de intercambio. « ¿De veras estos bienes no tienen otras cualidades comunes como puede ser su rareza en proporción a la demanda?», es decir la cualidad de presentarse en cantidades insuficientes para satisfacer todas las necesidades que de ellas tiene el ser humano, o «la de haber sido apropiadas por el hombre» precisamente por esa causa, o «la de ser objeto de oferta y demanda», se pregunta BöhmBawerk. Decídalo el lector.

Marx sigue con sus delirios: «si los bienes que son intercambiados sólo tienen en común la cualidad de ser productos del trabajo, entonces el valor de cambio vendrá determinado por la cantidad de trabajo incorporado en la mercancía». Marx descarta las «excepciones» como algo insignificante.

Böhm-Bawek examina esas "pocas excepciones sin importancia». Al final vemos que éstas predominan de tal modo que apenas dejan margen a la «regla» Se incluirían por ejemplo, los 
bienes que no pueden reproducirse a voluntad como por ejemplo las obras de arte y las antigüedades, toda la propiedad inmueble (¿Cómo explica Marx que un piso de 150 metros cuadrados, construido por los mismos obreros con los mismos materiales, en la calle Serrano de Madrid valga veinte veces más que el mismo piso en una pedanía de la provincia de Teruel?), los productos protegidos por patente o derechos de autor o los vinos de calidad (las horas de trabajo utilizadas para obtener vino de Vega Sicilia son más o menos las mismas que se emplean en producir un vino peleón cien veces más barato). ¿Y qué decir de los productos objeto de trabajo cualificado, provenga esta cualificación de la preparación profesional o de las dotes innatas? Aunque Marx sostenga que ésta última no es una excepción, sino una variante pues según él, «el trabajo complejo es trabajo simple potenciado o multiplicado», Böhm-Bawerk advierte que para explicar la realidad no interesa lo que los hombres puedan fingir que es, sino lo que real y verdaderamente es. ¿Puede alguien en su sano juicio sostener de verdad que dos horas de trabajo de un cantante de opera tienen idéntica esencia que sesenta horas de trabajo de un enfermero?

He dejado para el final la última gran excepción. Una excepción de tal calibre que en la actualidad incluye al 95 por ciento de los bienes. Se trata de todas aquellas mercancías producidas con el concurso de capital o, por mejor decirlo aquellos bienes en los que el tiempo ha jugado un papel importante en el proceso de su producción. Puesto que es sobre estos bienes sobre los que Marx construye su teoría de la plusvalía - considera que estos bienes no constituyen una excepción, sino la confirmación de la explotación capitalista- vamos a examinarlos con detalle.

\section{III LA «PLUSVALÍA» CAPITALISTA}

Para Marx, tanto el beneficio, como el interés del capital provienen de la explotación del trabajador. Veamos como trata de probarlo. Como hemos visto, Marx mantiene por un lado que los bienes se cambian en el mercado según el trabajo que llevan 
incorporado - lo cual se ha probado que es falso-, pero como según él el trabajador no recibe el producto íntegro de su trabajo - la segunda tesis que también demostraremos falsa-, sino tan sólo el mínimo salario de subsistencia, el capitalista puede apropiarse del excedente producido. Dice Marx: «El precio medio del trabajo asalariado es el minimo del salario, es decir, la suma de los medios de existencia de que tiene necesidad el obrero para seguir vivo como obrero. Por consiguiente, lo que el obrero recibe por su actividad es estrictamente lo que necesita para mantener su mísera existencia y reproducirla».

Para respaldar esta segunda tesis Marx apela al prestigio de la Escuela Clásica. Marx cita a Adam Smith:

«En el estado original de cosas, que precede tanto a la apropiación de la tierra como a la acumulación de capital, el producto integro del trabajo pertenece al trabajador. No existen ni terratenientes, ni patrón con quienes compartir.

Si hubiese continuado este estado de cosas, los salarios de los trabajadores habrían aumentado con todas las mejoras de la productividad a que la división del trabajo da lugar».

Marx también invoca la «ley de hierro de los salarios» avanzada por David Ricardo y refrendada por Lasalle. Para Ricardo, los salarios no pueden elevarse permanentemente por encima del nivel de subsistencia, ya que en tal caso se produce un incremento de población. Esto obliga a cultivar tierras cada vez menos fértiles con lo que se eleva el coste de producción del cereal - medio de subsistencia por antonomasia del obrero y base de toda la teoría ricardiana de la renta-.

Finalmente Marx se refiere a la teoría clásica según la cual el valor de cambio o precio, coincide con el coste de producción. Según Marx el coste de producción del trabajo es el coste de subsistencia del trabajador. El origen de la plusvalía radicaría pues en «la diferencia entre el coste de la fuerza de trabajo y el valor que ésta puede crear». Es decir, el obrero trabaja diez horas, pero sólo cobra lo producido en dos. De las otras ocho se apodera el capitalista. 


\section{IV}

\section{CRÍTICA DE LA TEORÍA DE LA PLUSVALÍA}

Vamos a examinar a continuación las principales falacias incluidas en estos últimos argumentos.

Aunque Böhm-Bawerk no se detiene a criticar la afirmación de Smith -incluso aceptando este marco teórico, demostró la falsedad de la teoría de la explotación y explicó el verdadero fundamento del interés del capital- Nosotros sí vamos a mostrar la doble falsedad que se esconde en la aseveración según la cual el salario sería la forma original y primaria de ingreso de la que emergería el beneficio como resta de aquel.

Primero: si definimos salario como la retribución al trabajo dependiente (la definición que Marx siempre utiliza), es imposible que éste exista en la etapa pre-capitalista. El salario surge con el capitalismo. Los ingresos que los «trabajadores» percibían anteriormente - por ejemplo en el caso de granjeros o artesanos- no eran salarios, sino beneficio empresarial en la terminología marxista, pues eran los propietarios de la producción los que vendían ésta en el mercado, los que organizaban el proceso productivo y los que eran dueños de los instrumentos materiales que hacían posible la misma. Lo mismo cabe decir de los comerciantes que compraban mercancía para revenderla con beneficio. Es evidente que cuando se compra mercancía no se paga salario y que tampoco se cobra cuando se vende. Los comerciantes compraban capital constante en la terminología marxista que luego explicaré y éste como veremos no puede producir beneficio.

Segundo: Smith, igual que Marx, desprecia e ignora absolutamente los efectos absolutamente decisivos que, para la división del trabajo y el incremento de la productividad, tienen la propiedad privada, la acumulación de capital y la función empresarial. En realidad la «época dorada» a la que parece referirse Smith sería el paleolítico, en donde hordas de salvajes subhumanos se dedicaban exclusivamente a la depredación - caza y recolección-, sin que existiese nada parecido a una transformación de recursos en etapas sucesivas para lograr bienes distintos de los que ofrecía la naturaleza en estado salvaje. La revolución neolítica que introduce el cultivo agrícola y la ganadería 
y que eleva al primate a la condición de hombre, se basó en una institución fundamental: la propiedad privada.

Por lo que a la ley de hierro de los salarios se refiere, ésta no se basaba tanto en el hecho de que los trabajadores son explotados (por tanto queda fuera del análisis de Böhm-Bawerk) y no perciben íntegramente el fruto de su trabajo - Ricardo no parece compartir esta tesis-, sino en la aplicación combinada de dos principios: la ley de los rendimientos marginales decrecientes en la agricultura y las ideas que sobre el crecimiento de la población había avanzado Thomas Malthus: «la población de los seres vivos tiende a expandirse hasta el límite en el que los recursos disponibles no pueden garantizar más que el mínimo de subsistencia». Estas ideas que han sido refutadas por la realidad en todos los países de Occidente, también han sido contestadas en el campo teórico.

La ley de los rendimientos marginales decrecientees establece que si se aumenta la cantidad empleada de un factor de producción, manteniéndose constantes las cantidades empleadas del resto de factores, la cantidad producida, aumenta, a partir de cierto momento, en proporciones cada vez menores. Es verdad que existe una ley de rendimientos marginales decrecientes, no sólo en la agricultura, sino en todos los campos de la producción (si no existiese o toda la producción se concentraría en un metro cuadrado, o no haría falta acumular capital o todo el trabajo del mundo podría ser realizado por un solo operario), pero - y esto es lo importante- dicha ley convive con otro principio económico como es que la división del conocimiento y la acumulación de capital mejoran las técnicas de producción e incrementan por tanto la productividad. Hayek tenía mucha razón cuando decía que debemos optar entre ser pocos y pobres o muchos y ricos. Es difícil determinar cuál es el volumen óptimo de población en cada momento, aunque advertimos que los seres humanos son bastante racionales - a diferencia de los animales - a la hora de regular la población, mediante lo que se conoce como paternidad responsable, es decir, no echar al mundo hijos a los que no se tenga la oportunidad de proporcionar una vida al menos tan cómoda como la que disfrutan los padres. ¡Si Marx creía que los trabajadores iban a comportarse como animales y no como humanos a la hora de reproducirse, no parece que les tuviera en muy alta estima! 


\section{VALOR Y COSTE DE PRODUCCIÓN}

Es la idea de que el coste de producción determina el valor de cambio o precio del producto sobre la que Bóhm-Bawerk recrudece sus críticas.

Como decía Jim Cox si el valor de los bienes estuviese determinado por su coste de producción, la foto de un ser querido tendría el mismo valor que la de un desconocido o la de un enemigo - abran sus carteras para comprobarlo-. Me pregunto que hacen dos marxistas después de ir al cine. Se supone que no podrán estar en desacuerdo sobre lo mucho o poco que les ha gustado la película, pues después de todo, la producción ha requerido igual cantidad de trabajo antes de que ambos la consuman.

En realidad, ninguna actividad de tipo industrial o de cualquier otro orden, puede conferir valor al bien o servicio producido. El valor brota posteriormente de las apreciaciones subjetivas de la gente. Es la intensidad de la apetencia del consumidor la que determina el valor de bienes y servicios. Es importante subrayar que lo que el consumidor valora no es la totalidad de bienes que existen en el universo (todo el agua o el pan del mundo), sino solamente la unidad o unidades (una botella, una barra) sobre los que ha de decidir. Los que puede o no adquirir y los que puede o no ceder a cambio.

A partir de esta genial observación - a nosotros nos parece evidente una vez presentada-, Menger y luego Böhm-Bawerk construyen una completa teoría de precios y costes. Si los bienes de consumo se valoran de acuerdo con la necesidad que satisface o deja de satisfacer la unidad de cada bien sobre la que tenemos que decidir, los factores de producción se valoran según su aptitud para proporcionarnos aquellos bienes, esto es según su productividad. Aquí también hablamos de unidades concretas y «marginales» (están en el margen de ser o no adquiridas o cedidas) y no de la totalidad del factor que existe en el mundo. Cada unidad de factor es valorada así de acuerdo con su productividad marginal.

La Ciencia Económica tradicionalmente había clasificado los factores de producción en tres grandes grupos: tierra, trabajo y capital. La genial aportación de Bóhm-Bawerk consistió en 
mediante el análisis de un factor ignorado, el tiempo, descubrir la auténtica esencia del capital.

Veamos como utiliza el austríaco el tiempo, para ridiculizar la teoría de la explotación. Una cosa es que deba pertenecer al obrero el producto íntegro de su trabajo o su valor correspondiente - lo cual Bóhm-Bawerk y cualquiera acepta-y otra que el obrero deba percibir ahora todo el valor futuro de su trabajo. Los socialistas pretenden, por llamar a las cosas por su nombre, que los obreros perciban a través del contrato de trabajo más de lo que producen, más de lo que obtendrían si trabajasen por cuenta propia. Böhm-Bawek ilustra el argumento con algunos ejemplos:

«Imaginemos que la producción de un bien, por ejemplo de una máquina de vapor, cueste cinco años de trabajo, que el valor de cambio obtenido de la máquina terminada sea 5.500 florines y que intervengan en la fabricación de la máquina cinco obreros distintos, cada uno de los cuales ejecuta el trabajo de un año. Por ejemplo, que un obrero minero extraiga durante un año el mineral de hierro necesario para la construcción de la máquina, que el segundo dedique otro año a convertir ese mineral en hierro, el tercero a convertir el hierro en acero, que el cuarto fabrique las piezas necesarias y el quinto las monte y dé los toques finales a ésta. Según la naturaleza misma de la cosa, los cinco años de trabajo de nuestros obreros no podrán rendirse simultánea, sino sucesivamente y cada uno de los siguientes obreros sólo puede comenzar su trabajo una vez hayan culminado el suyo los obreros anteriores. ¿Qué parte podrá reclamar por su trabajo cada uno de los cinco copartícipes, con arreglo a la tesis de que el obrero debe percibir el producto integro de su trabajo?

Si no existe un sexto elemento extraño que anticipe las retribuciones, deberán tenerse en cuenta dos puntos absolutamente seguros. El primero es que no podrá efectuarse el reparto hasta pasados cinco años. El segundo es que los obreros pueden repartirse los 5.500 florines. Pero, ¿con arreglo a qué criterio? No por partes iguales, como a primera vista pudiera parecer, pues ello redundaría considerablemente a favor de aquellos obreros cuyo trabajo corresponde a una fase posterior del proceso productivo y en perjuicio de los que han aportado su trabajo en una fase anterior. El obrero que monta la máquina percibiría 1.100 florines por su año de trabajo inmediatamente después de terminado 
éste; mientras, el minero no obtendría su retribución hasta pasados cuatro años. $Y$ como este orden de preferencia no puede ser en modo alguno indiferente a los interesados, todos ellos preferirían el trabajo final y nadie querría hacerse cargo de los trabajos iniciales. Para encontrar quien aceptase éstos, los obreros de las fases finales se verían obligados a ofrecer una participación más alta a sus compañeros encargados de los trabajos preparatorios. La cuantía de esta compensación dependería de dos factores: la duración del aplazamiento y la magnitud de la diferencia que existe entre la valoración de los bienes presentes y futuros. Así por ejemplo si esta diferencia fuese del 5 por ciento anual, las participaciones se graduarían: 1.200 florines para el primer obrero, 1.150 para el segundo, 1.100 para el tercero, 1.050 para el cuarto y 1.000 para el quinto.

Sólo podría admitirse la posibilidad de que los cinco cobrasen la misma suma de 1.100 florines partiendo del supuesto de que la diferencia de tiempo les fuese indiferente.»

Pero, si realmente el tiempo fuera indiferente a la hora de determinar el valor y por tanto la cuantía de la retribución, a los obreros les daría igual cobrar el día siguiente a la terminación de su tarea que transcurridos cinco años y, si esto fuera así, les daría igual cobrar a los cinco años que pasados cincuenta, cien o mil. (No me cabe duda de que todos los empresarios subirían muy generosamente los sueldos a quienes esperasen tan largo tiempo para cobrar). En realidad, el interés no es la retribución por la abstinencia - la tesis de Nassau Senior ridiculizada por Lasalle-, ni la apropiación del trabajo del obrero - como dicen los socialistas-, sino la manifestación en el mercado de un presupuesto de la acción humana, a saber, que los seres humanos pretenden alcanzar sus fines cuanto antes. De no ser así, se optaría siempre por los procesos más productivos cualquiera que fuese el tiempo que estos requiriesen hasta completarse, llegándose a un punto en que desapareciese la producción de bienes de consumo, pues toda los factores se emplearían en investigación, desarrollo y acumulación de capital.

Seguimos con el ejemplo: "Supongamos ahora que los obreros, como ocurre en la realidad, no puedan o no quieran esperar para recibir su salario a que termine el proceso productivo y que entren en tratos con un empresario para obtener de él un salario a medida que 
vaya rindiendo su trabajo, a cambio de lo cual el empresario adquiere la propiedad del producto. Supongamos que este empresario sea una persona exenta de todo sentimiento egoísta. (...) ¿En qué condiciones se establecería el contrato de trabajo? No cabe duda de que el trato por los obreros sería absolutamente justo si el empresario les paga como salario exactamente lo mismo que recibirían como parte alícuota en el caso de organizar la producción directamente y por cuenta propia. En este caso 1.000 florines inmediatamente después de terminar su trabajo, que era lo que percibía el obrero que cobraba inmediatamente. Puesto que los cinco obreros aportan exactamente el mismo trabajo, lo justo será que perciban el mismo salario».

Existen otros ejemplos aún más contundentes. Supongamos que un vino necesita madurar en la barrica durante veinte o cuarenta años para alcanzar una calidad extraordinaria. Los cultivadores, recolectores y pisadores de la uva, no pueden cobrar hasta pasadas decenas de años salvo que un capitalista les adelante su retribución. Pero, si quieren cobrar inmediatamente después de finalizar su tarea, deberán hacerlo no conforme al valor del vino ya maduro, sino de acuerdo al valor del vino sin edad que es notablemente inferior. Si alguien les anticipa sus retribuciones y luego vende el vino pasados cuarenta años, ¿de verdad creen los socialistas que dicho empleador debe buscar a sus antiguos operarios y retribuirles con los intereses del capital? Y si el vino se malogra o cae de valor debido a cambios en el gusto de los consumidores, ¿tendría sentido que les persiguiese para exigirles el reembolso de lo cobrado?

VI

\section{CAPITAL CONSTANTE Y CAPITAL VARIABLE}

Marx decía que el beneficio y el interés capitalista procedían del trabajo prestado y no retribuido. Por tanto, la composición del coste de producción era determinante a la hora de determinar el rendimiento del capital. Según Marx, sólo el capital empleado en pagar salarios a los trabajadores podía producir beneficio. Marx llamó a esta parte, capital variable; era variable porque crecía merced a la explotación de los obreros. Por su 
parte el dinero empleado en adquirir materiales y maquinaria no era capaz de generar plusvalía. Hay que recordar que éstos ya se habrían vendido según el trabajo incorporado, dejando la plusvalía en poder del vendedor. Marx llamó a esta parte, capital constante.

Marx se apartaba por tanto de la teoría económica clásica que sostenía que la tasa de rendimiento del capital tendía a ser constante cualquiera que fuese su composición. Puesto que los clásicos - Smith, Ricardo, Mill - propugnaban la teoría del valor derivado del coste de producción, su fórmula determinante del valor era: capital constante + capital variable + tasa de rendimiento medio. (En realidad Menger y Böhm-Bawerk habían demostrado que la causalidad iba en sentido inverso. Los costes de los factores se formaban a partir del precio que se esperaba obtener.)

La gran innovación del primer volumen de El Capital era pues, la nueva fórmula del precio de equilibrio: capital constante + capital variable + plusvalía, siendo ésta última mayor o menor según el porcentaje relativo de capital variable respecto de capital fijo. Dicho de otra forma, cuantos más obreros y menos máquinas interviniesen en la producción mayor beneficio se obtenía y viceversa. De este principio Marx deducía su teoría de la crisis capitalista, más y más aguda conforme crece la acumulación de capital y caen los beneficios.

Sin embargo, ya vimos que Marx se daba cuenta de que su fórmula no se veía refrendada por la realidad. En una huida hacia delante, calificó esta contradicción de «aparente» y prometió resolverla en el tercer volumen. Aunque Marx falleció sin publicarlo, Engels sí lo hizo a partir de su manuscrito. Como dice Böhm-Baweerk la aparición de este volumen era esperada con cierta expectación en los círculos teóricos de todos los partidos, para ver como Marx se las iba a arreglar para resolver un problema que en el primer volumen ni siquiera había tocado.

Pues bien, en el tercer volumen, Marx reconoce expresamente que en la realidad, gracias a la acción de la competencia, las tasas de ganancia del capital, cualquiera que sea su composición, se mueven sobre la base de un porcentaje igual de ganancia media. Marx dice: «En la vida real las mercancías no se cambian de acuerdo con sus valores (sic), sino con arreglo a sus precios de 
producción». Es decir, las mercancías equiparadas por medio del intercambio contienen real y normalmente cantidades desiguales de trabajo. ¿Cabe mayor retractación? La fórmula en el tercer volumen vuelve a ser la de los clásicos: capital constante + capital variable + tasa media de beneficio. Por tanto, aunque Marx no lo diga, carece ya de sentido la fantasmagórica distinción entre capital constante y variable. De igual modo, no queda sitio para el supuesto colapso debido a la excesiva acumulación de capital no rentable. ¿Y como justifica Marx tal contradicción? Simplemente la niega:

Marx dice más o menos: «Es cierto que las distintas mercancías se cambian unas veces por más de su valor y otras veces por menos, pero estas divergencias se compensan o destruyen mutuamente, de tal modo que, tomadas todas las mercancías cambiadas en su conjunto, la suma de los precios pagados es siempre igual a la suma de sus valores. De este modo, si nos fijamos en la totalidad de las ramas de producción tenemos que la ley del valor se impone como 'tendencia dominante.»

La respuesta de Böhm-Bawerk merece ser reproducida con cierta extensión, pues nos muestra a éste en su mejor forma: "¿Cuál es en realidad, la función de la ley del valor? No creemos que pueda ser otra que la de explicar las relaciones de cambio observadas en la realidad. Se trata de saber por qué en el cambio, por ejemplo, una chaqueta vale veinte varas de lienzo, por qué diez libras de té vale media tonelada de hierro, etc. (...) Tan pronto como se toman todas las mercancías en su conjunto y se suman sus precios se prescinde forzosamente de la relación existente dentro de esa totalidad. Las diferencias relativas de los precios entre las distintas mercancías se compensan en la suma total. (...) Es exactamente lo mismo que si a quien preguntara con cuantos minutos o segundos de diferencia ha llegado a la meta el campeón de una carrera con respecto a los otros corredores se le contestara que todos los corredores juntos han empleado veinticinco minutos y treinta segundos. (...) Por ese mismo procedimiento podría comprobarse cualquier "ley", por absurda que fuera, por ejemplo, la "ley" de que los bienes se cambian de acuerdo a su peso especifico. Pues aunque en realidad una libra de oro, como "mercancía suelta", no se cambia precisamente por una libra, sino 
por 40.000 libras de hierro, no cabe duda de que la suma de los precios que se pagan por una libra de oro y 40.000 libras de hierro tomadas en su conjunto, corresponden exactamente a 40.0000 libras de hierro más una libra de oro. La suma de los precios de las 40.001 libras corresponderá pues, exactamente al peso total de 40.001 libras materializado en la suma de valor, por donde, según aquel razonamiento tautológico, podremos llegar a la conclusión de que el peso es la verdadera pauta con arreglo a la cual se regula la relación de cambio de los bienes.

La realidad es la siguiente. Ante el problema del valor, los marxistas empiezan contestando con su ley del valor, consistente en que las mercancías se cambian en proporción al trabajo materializado en ellas. Pero más tarde revocan esta respuesta —abierta o solapadamente-en lo que se refiere al cambio de las mercancías sueltas, es decir, con respecto al único campo en que el problema del valor tiene un sentido, y sólo la mantienen en pie, en toda su pureza, respecto al producto nacional tomado en su conjunto, es decir con respecto a un terreno en el que aquel problema no tiene sentido alguno. Lo cual equivale a decir tanto como reconocer que, en lo tocante al verdadero problema del valor, la "ley del valor" es desmentida por los hechos; en la única aplicación en que los hechos no la desmienten, no constituyen ninguna respuesta al verdadero problema.»

\section{VII CONCLUSIÓN}

La refutación de Böhm-Bawerk a la teoría de la explotación constituye, como decía Rothbard, la vacuna que, por excelencia, inmuniza contra el marxismo. Sobre ella lanzaron los marxistas, primero sus más furibundos ataques, - en realidad contra su «lógica burguesa» ya que los argumentos son incontrovertibles-. Ahí están los trabajos de Hilferding, Bujarin o Sweezy para quien quiera reír, por no llorar. Más adelante, simplemente lo silenciaron. Ese silencio ha llevado desgraciadamente a que cientos de millones de personas hayan sufrido y sigan sufriendo la opresión de tiranos comunistas que venden humo, engendran odio y fabrican miseria. Esperemos que este trabajo aporte su grano de arena para revertir esa tendencia. 


\section{REFERENCIAS BIBLIOGRÁFICAS}

von BÖHM BAWERK, Eugen (1987). La Teoría de la explotación, capítulo incluido dentro de Historia y crítica de las teorías del interés (Ed. Fondo de Cultura Económica). Existe una edición separada de Unión Editorial que está agotada, pero que aparece incluida dentro del Volumen III de Lecturas de Economía Política de Jesús Huerta de Soto (Unión Editorial).

- (2000). La Conclusión del Sistema marxiano (Unión Editorial).

- (1988). La Teoría Positiva del Capital (Ediciones Aosta).

MARX, KARL (1947). El Capital. Tres volumenes (Fondo de Cultura Económica). 